\title{
Libro: Buen vivir y descolonialidad. Crítica al desarrollo y la racionalidad instrumentales
}

Boris Marañón Pimentel (coordinador)

UNAM, IIE, México, 2014, (267 pp.)

\section{Sebastián Pérez}

Licenciado en Ciencia Política, Facultad de Ciencia Política y Relaciones Internacionales, Universidad Nacional de Rosario, Argentina.

E-mail: sebastian_perez34@hotmail.com

El ejercicio de pensar desarrollos alternativos que reincorporen la integralidad de la vida humana supondría un mayor esfuerzo que el de adscribir a perspectivas sistémicas aficionadas a la dimensionalización/destazado de la vida. Sucede que en realidad el esfuerzo no es mayor sino que es de otro carácter, de otra naturaleza. Esto es "Buen vivir y descolonialidad. Crítica al desarrollo y la racionalidad instrumentales". La obra, publicada en febrero de 2014, se constituye en un esfuerzo colectivo por pensar el desarrollo, las actividades humanas en dirección de su crecimiento y auto-superación subjetiva, desde lógicas, que conscientemente buscan colocarse por fuera de la racionalidad instrumental capitalista y eurocéntrica. Ejercicio de resistencia, este último, vital en la fase capitalista neo-extractiva que actualmente transitamos.

La obra compilada por Boris Marañón Pimentel se inscribe institucionalmente en el ámbito que mayor desarrollo muestra en las temáticas tratadas, la Universidad Nacional Autónoma de México, en particular su Instituto de Investigaciones Económicas. Presenta explícitamente dos partes en las que se desarrollan, por un lado, los aspectos teóricos que asistirán a los autores y, por otro, una segunda parte que camina la senda del discurso a la práctica. Se pretende avanzar en aspectos teórico-conceptuales y de análisis de casos para indagar las condiciones de un nuevo imaginario histórico anticapitalista. Se ponen en discusión los elementos teóricos de racionalidades alternativas diferenciadas y opuestas a las hegemónicas.

Desde su introducción se identifica como perspectiva epistemológica la Teoría de la colonialidad/descolonialidad del poder, iniciada por Aníbal Quijano. El Buen Vivir es reconocido, desde este marco epistemológico, como "una alternativa que puede contribuir a restablecer un lazo relacional entre las personas y con la naturaleza, basado en la solidaridad y la reciprocidad" (pp. 10-11). Articulando lo que la obra define como la Razón Histórica Moderna y la Razón India, el Buen Vivir es posicionado como alternativa solidaria y liberadora, ejercicio integral de descolonización que sobrevolará todo el libro. La primera parte de la obra buscará, como dijimos, discutir los elementos teóricos y sumar aportes a una propuesta analítica; la segunda apuntará a estudios de caso que respondan a la pregunta de 
investigación, quizá más valiosa, “¿cómo se procesa esa nueva Racionalidad en la vida cotidiana, con que contracciones y avances?” (p.12).

El primer capítulo, a cargo de Marañón Pimentel, es una continuidad de la introducción ya que adquiere un carácter integrador de la perspectiva teórica cerrando con una enumeración de conceptos que recorrerán toda la compilación.

La Teoría de la colonialidad es presentada desde el asentamiento de las lógicas capitalistas "en una estructura de explotación y dominación en la que la clasificación social de la población mundial tendrá su principal sustento en la idea de raza; esto es, en la construcción-imposición de diferencias sociales entre la gente a partir de sus diferencias biológicas (color de la piel), lo que se constituirá en el criterio de clasificación social en la modernidad/ colonialidad, para la distribución de la población en las relaciones de poder (p. 23). Se supone así que lo moderno, lo colonial y lo capitalista forman parte de un mismo proceso histórico. No corresponde aquí seguir desarrollando la teoría iniciada por Quijano, pero al capítulo este repaso le permite presentar conceptos centrales de la obra como la racionalidad instrumental, venida de la mano del eurocentrismo como pretendida perspectiva científica universal. Desde allí supone el crecimiento y desarrollo de una/unas racionalidad/es alternativas. Allí entrarán en escena las experiencias basadas en el Buen Vivir como concepción articuladora de experiencias de resistencia colectiva.

En medio de toda una presentación teórica acompañada de un análisis crítico constante del eurocentrismo y la racionalidad instrumental, quizá son dos los intereses más relevantes que presenta este capítulo. El primero es el enmarque de la conceptualización en contextos de desempleo estructural, financiarización e hipertecnocratización que completan el cuadro explicativo. En segundo lugar, la idea rectora del pasaje de una racionalidad instrumental a una alternativa se basaría aquí en el abandono de las perspectivas sujeto-objeto hacia otras de sujeto-sujeto. En esta última se reconoce a la naturaleza, los bienes comunes, a la Madre Tierra con derechos por sí misma. Esto supone la dislocación más relevante quizá del paradigma antropocéntrico del eurocentrismo.

En el capítulo segundo se abandona la pretensión de integralidad explícita. Carla Beatriz Zamora Lomelí intenta abordar más específicamente una crítica a las perspectivas hegemónicas/sistémicas de la movilización social para un-simultáneo- reconocimiento de una perspectiva diferenciada en la colonialidad. Se afirma que las perspectivas de la anglosajona movilización de recursos y francesa de los nuevos movimientos sociales "se basan en principios de exclusión sistémica" (p. 63). Con todo se mantendría al colectivo con aspiraciones para incorporarse/ incluirse a un esquema de poder dado. La autora hablará de una perspectiva crítica en la cual "no consideran las relaciones de dominio-subordinación que prevalecen en una sociedad estratificada, o cuestiones como la resistencia de los grupos dominados y la construcción de alternativas frente al capital, como ocurre a nivel local y global en la práctica de algunos movimientos sociales" (p. 63). Para ello ahondará en definiciones y dimensiones internas a los conceptos de movimiento social y acción colectiva para ejercitar la diferenciación de una racionalidad li- 
beradora y solidaria que le permita plantear alternativas frente a las relaciones de dominio-subordinación.

Sergio Tischler y Mina Lorena Navarro analizan la dimensión de oposición entre comunidad y capital. Todo este capítulo tercero aborda la mercancía, el fetichismo, el valor con un gran anclaje marxista clásico. La oposición de comunidad abstracta frente a comunidad humana, emparentada íntimamente con la anterior de comunidad-capital, es tratada desde el descentramiento de las necesidades humanas que producen las lógicas de la mercancía. Todo esto, claro, como técnica de dominación del capital. La resistencia viene dada entonces desde la reactualización de temas comunitarios. Desde aquí se define a la lucha socioambiental, ahondando en una caracterización que complementa el desarrollo del capítulo anterior.

El cuarto capítulo, atendiendo al -y munido del- ejercicio crítico realizado por ya más de un tercio de la obra, se arroja de forma más directa a afirmar una propuesta de reciprocidad/solidaridad. Quizá lo más interesante aquí sea la presentación de la reciprocidad como lógica comunitaria previa, simultánea y superviviente a las lógicas del capital. El propio capitalismo es definido desde su capacidad de subordinación de las lógicas alternativas con las que se encuentra en su avanzada. La definición de reciprocidad es realizada desde una gran multiplicidad de perspectivas. Incluso llegan a considerarse perspectivas alternativas del primer mundo, como el decrecimiento o las perspectivas antiutilitaristas.

El capítulo cinco a cargo de Tirso Gonzales, aborda directamente el Kawsay (Buen Vivir) y la afirmación cultural. La perspectiva andina del Pratec-Naca, que desarrolla, le permite afirmar que la sustentabilidad y la agroecología como objetivos de resistencia requieren tener en cuenta la "intelligentsia indígena contemporánea" (p. 135). Esta perspectiva indígena-campesina es presentada como paradigma de epistemología alternativa, como ejercicio de descolonización del pensamiento. Cierra recomendando la articulación de institutos de investigación autónomos con las organizaciones campesinas, ejercicio de reindigenización de la alternatividad.

José Gasca Zamora inaugura la segunda parte del libro, abocado al análisis de casos desde las conceptualizaciones más arriba señaladas. En su artículo se detienen a analizar la estructuración de las decisiones en el ámbito de las comunidades, para abordar específicamente una experiencia de administración comunal de recursos naturales del bosque en la Sierra Norte de Oaxaca, México. Como en el resto de los análisis de caso aquí se ponen claramente en evidencia las tensiones que genera la colisión de articulaciones y formas de trabajo internas a la comunidad o empresa comunal basadas en la reciprocidad, y las lógicas de mercado a las cuales acuden para colocar sus productos. Sobre estas tensiones y, sobre todo, sobre la idea de comunalidad materializada en el emprendimiento silvícola analizado versa el artículo que ocupa el capítulo sexto de nuestra obra.

Rosendo Montiel y Leónides Oliva, en el capítulo séptimo, desarrollarán la idea de economía solidaria como apropiación integral del proceso productivo por parte de la comunidad desde cuatro pilares propios: el territorio, el tequio, la fiesta y la asamblea. El caso analizado son las Comunidades Campesinas de Camino, en un 
proceso de asunción directa de todo el ciclo productivo y de comercialización de diversos productos bajo lógicas comunitarias.

Marín Rubio López y Blanca Rubio Pacheco nos comparten la experiencia cooperativista de Unidad, Desarrollo y Compromiso, con sede en Anenecuilco, Ayala, Morelos, de la que se presentan como socios fundadores. En este octavo capítulo los autores ponen de manifiesto las fuentes de inspiración de este emprendimiento, el Cooperativismo integral y los pioneros de Rochdale. La dinámica comunitaria se ve, en este apartado, desdibujada por lógicas de ahorro, consumo y producción que operan otro tipo de anticapitalismo. En particular esto se ve en la idea de la incorporación de los denominados agiotistas, entendiendo que en la resolución de los problemas cotidianos de cada quien se produce un primer encuentro para iniciar un proceso de educación de los miembros que vaya en dirección de un creciente compromiso solidario. Se habla aquí de cambio de hábitos.

El capítulo noveno retoma la cuestión de las economías solidarias afirmándolas como la integración de dos subprocesos, uno económico y otro educativo. Aquí los autores, coordinados por Mario Bladimir Monroy Gómez, ponen en debate la experiencia educativa del Instituto Intercultural Ñöñho, de Aguas Calientes del cual son profesores, en particular su Licenciatura en Emprendimientos en Economía Solidaria. Con un detallado recuento histórico se llega a esta institución que tiene como metas la apertura de la educación superior a la Comunidades Indígenas, y el desarrollo de proyectos y soluciones a los problemas del y desde el territorio. Se piensa la experiencia del Instituto en consonancia con tesis de Dussel y Fannon, citados en el trabajo. El instituto significa así un esfuerzo más por el perfeccionamiento de las experiencias sociales económicas y políticas, que presentan a la Solidaridad como vertebrador de comportamiento económico y de la vida.

El apartado siguiente realiza una interesante aplicación de la teoría de la colonialidad sobre la idea de Hábitat. La idea rectora de gestión social del hábitat, en oposición a la mercantilización de la vida, y su percepción subjetiva por la misma comunidad que la aplica estructuran este decimo capítulo. Ninett Torres Villarreal nos introduce en la rica experiencia de la Cooperativa de Vivienda Palo Alto, con el interés puesto en la perspectiva de los actores que la construyeron. Nuevamente el cooperativismo es señalado como promotor de (y/o mecanismo para) una racionalidad de reciprocidad y solidaridad frente a la hegemónica racionalidad instrumental. El capítulo retoma un esfuerzo conceptual y de repaso teórico no visto desde el capítulo quinto de la compilación. Retorna, claro, a la colonialidad para abordar el caso de Palo Alto. Se preocupa por observar la gestación de racionalidades alternativas desde un análisis de las percepciones subjetivas de los participantes en el proceso.

El último capítulo aborda el caso de la Cooperativa de Trabajadores Democráticos de Occidente (Tradoc ex Euzcadi), en el Salto, Jalisco, México. Sarya Luna Broda nos propone un recorrido en que se antepone el derecho al trabajo por sobre el derecho de propiedad. La autogestión puesta en juego por esta experiencia de empresa recuperada por sus trabajadores permite comprender que el trabajo, en este ámbito, puede entenderse no sólo desde su carácter de explotación/do- 
minación sino como forma de relación social dada a la realización colectiva. Se evidencian así el nacimiento y desarrollo, reconocidos por la autora, de formas de producción y organización del trabajo alternativas.

Buen vivir y descolonialidad. Crítica al desarrollo y la racionalidad instrumentales significa un gran aporte para el campo de estudio que toca, en sentido de articulación conceptual y de recolección de experiencias. En general la obra presenta las debilidades de su tipo. Al ser un trabajo colectivo, por más esfuerzo de compilación y coordinación, los artículos del primer apartado teórico acometen el esfuerzo de definir varias veces el mismo concepto. La operación es reconocible en algunos artículos de análisis de casos en que los conceptos de reciprocidad, colonialidad, solidaridad, racionalidad instrumental son re-abordados, para el gusto de este lector, de manera un tanto repetitiva. Desde una mirada positiva, puede entenderse, por lo dicho antes, que la repetición habla también de la claridad y la concordia conceptual de los escritores. Más aún si tenemos en cuenta que los casos analizados en la segunda parte del libro abordan experiencias campesino-indígenas, de vivienda urbanas, cooperativistas de producción, educación y trabajo, con diversas, también, fuentes filosóficas desde las cuales pensar una racionalidad liberadora, recíproca y solidaria. El abultado glosario que corona la obra, creemos, apunta a reintegrar aspectos conceptuales que a simple vista parecerían dispares. Sin temor a decirlo, creemos que el libro realiza operaciones de articulación conceptual y aplicación teórica que lo vuelven fundamental en el entendimiento de expresiones sociopolíticas del desarrollo alternativas. Operaciones que sería recomendable replicar. 